\title{
Síntese e caracterização de vermiculita mesoporosa obtida por modificação com sais complexos de alumínio e lantânio
}

\section{(Synthesis and characterization of mesoporous vermiculite obtained by modification with aluminum complex salts and lanthanum)}

\author{
M. V. S. Fernandes, L. R. D. da Silva \\ Laboratório de Pesquisa, Desenvolvimento e Inovação em Minerais Não-Metálicos, Universidade Federal do \\ Ceará, C.P. 6002, Campus do Pici, Fortaleza, CE, Brazil 60440-970 \\ lindomar@dqoi.ufc.br
}

\begin{abstract}
Resumo
Os estudos de aumento do espaçamento interplanar basal, distribuição de poros e área superficial de uma argila mesoporosa obtida a partir de vermiculita precursora, foram realizados por intercalação de oligômero poli-hidroxi catiônico $\mathrm{Al}_{13}$ e um misto (La $\left.\mathrm{Al}_{13-\mathrm{x}}\right)$ obtido por dopagem com lantânio, seguida de calcinação para formação dos pilares. As caracterizações das amostras foram feitas por difração de raios X, espectroscopia Mössbauer, ressonância magnética nuclear do estado sólido ( ${ }^{27}$ Al MAS-NMR) e isotermas de $\mathrm{N}_{2}$ (BET). A presença de lantânio na estrutura da vermiculita resultou no aumento de sua área e de porosidade.

Palavras-chave: lantânio, argila mesoporosa, ${ }^{27} \mathrm{Al}$ MAS-NMR, BET.
\end{abstract}

Abstract

The studies of increased basal interplanar spacing, pore distribution and surface area, mesoporous of a clay obtained from vermiculite precursor were obtained by intercalation of cationic polyhydroxy oligomer $A l_{13}$ and a mixture (La $\left.\mathrm{Al}_{13-x}\right)$ obtained by doping with lanthanum, followed by calcination to form the pillars. The characterizations of the samples were performed by $X$-ray diffraction, Mössbauer spectroscopy, nuclear magnetic resonance solid state ( ${ }^{7}$ Al MAS-NMR) and $N_{2}$ isotherms (BET). The presence of lanthanum in the structure of vermiculite resulted in the increase of its area and porosity.

Keywords: lanthanum, mesoporous clay, ${ }^{27} \mathrm{Al} M A S-N M R, B E T$.

\section{INTRODUÇÃO}

Demonstrou-se nas últimas décadas que as argilas têm um papel importante em diversos processos industriais [1]. Argilas como a vermiculita em particular são largamente utilizados na indústria, assim como em pesquisas básicas, tanto naturais ou em formas modificadas [2-5]. A vermiculita é um mineral lamelar formado pela alternância de folhas tetraédricas e octaédricas em uma razão 2:1 [68]. Caracteriza-se por um elevado grau de substituição isomórfica de $\mathrm{Si}^{4+}$ por $\mathrm{Al}^{3+}$ e/ou $\mathrm{Fe}^{3+}$ nas folhas tetraédricas, e $\mathrm{Mg}^{2+}$ por $\mathrm{Al}^{3+}, \mathrm{Fe}^{3+}$ e $\mathrm{Fe}^{2+}$ nas folhas octaédricas [9-13]. Acabam por serem sólidos ácidos muito atraentes com elevada estabilidade térmica superior em comparação com outras argilas semelhantes [14]. Devido a uma grande variedade de composições possíveis, a vermiculita apresenta uma elevada capacidade de troca cationica (CTC) de 100$150 \mathrm{meq} / 100 \mathrm{~g}$ de argila [15].

Materiais porosos podem ser aplicados em muitas áreas, na catálise heterogênea para adsorção de gases [16], o que torna a sua síntese de grande interesse para a comunidade científica. Os materiais porosos podem ser obtidos a partir de minerais de argilas combinando a utilização de agentes intercalantes e a pilarização [17]. Independentemente da via de síntese, os materiais adequados são aqueles que se encontram numa região de micro para mesoporosos [18].

Tratamentos físicos e/ou químicos (tais como troca catiônica, intercalação de compostos orgânicos e/ou sais complexos, acidificação, pilarização, lixiviação, etc.) têm a capacidade de modificar as propriedades texturais e estruturais das argilas, bem como acidez. A capacidade de adsorção de minerais de argila é influenciada por essas modificações [19]. Uma via comum para a síntese de materiais mesoporosos é a pilarização de argilas, onde são intercaladas com grandes cátions poliméricos $\left(\mathrm{Al}^{3+}\right.$, por exemplo), seguido de tratamentos hidrotermais, a fim de formar óxidos estáveis que atuam como pilares. Estes pilares evitam o colapso da estrutura, como resultado da expansão da argila, criando assim uma estrutura mesoporosa [20].

A capacidade de intercalar minerais de argila com vários óxidos ativos [21] revela a medida da importância destes materiais como argilas modificadas por pilares mistos, oferecendo um grande potencial como adsorventes e catalisadores em reações de vários tipos de interesse industrial [22,23]. Estrutura porosa e a química de superfície determinam o processo de adsorção, enquanto a distribuição de tamanho de poro afeta na adsorção a eficiência e seletividade [24]. Portanto, a microestrutura (distribuição de 
tamanho de poros, em particular) resultou do processo de pilarização que determina a utilidade desses materiais [25].

Neste trabalho, argilas mesoporosas foram sintetizadas por intercalação da vermiculita com oligômero de alumínio dopado com lantânio do tipo $\mathrm{La}_{x} \mathrm{Al}_{13-\mathrm{x}}$ (razão $\left[\mathrm{OH}^{-}\right] /\left[\mathrm{Al}^{3+}\right]$ $=2,4$, e $\mathrm{x}=0$ ou 1). BET, DRX, espectroscopia Mössbauer $\left({ }^{57} \mathrm{Fe}\right)$ e ressonância magnética nuclear do estado sólido $\left({ }^{27} \mathrm{Al}\right.$ MAS-NMR), foram utilizados para determinar a distribuição de tamanho de poro, área superficial, e espaço entre as camadas de $\mathrm{Fe}$ e $\mathrm{Al}$, coordenação do alumínio em função da dopagem do oligômero com lantânio.

\section{EXPERIMENTAL}

\section{Materiais}

Uma vermiculita fornecida pela UBM (União Brasileira de Mineração) teve sua constituição química determinada por fluorescência de raios X. A modificação química da vermiculita a Al-PILV teve como base estudos anteriores [26]. A dopagem do oligômero de alumínio com lantânio foi realizada a partir da mistura da solução de $\mathrm{NaOH} 0,2$ mol.L $\mathrm{L}^{-1}$ com soluções de $\mathrm{AlCl}_{3} \cdot 6 \mathrm{H}_{2} \mathrm{O} 0,2$ mol.L $\mathrm{L}^{-1}$ e $\mathrm{LaCl}_{3} \cdot 6 \mathrm{H}_{2} \mathrm{O}$ 0,2 mol. $\mathrm{L}^{-1}$ obtendo-se a relação $\mathrm{La}_{\mathrm{x}} \mathrm{Al}_{13-\mathrm{x}}$, podendo $\mathrm{x}$ ser igual a 0 ou 1 , para que a razão $\left[\mathrm{OH}^{-}\right] /\left[\mathrm{Al}^{3+}\right]$, seja mantida em 2,4. As amostras obtidas são descritas na Tabela I.

Tabela I - Descrição das amostras utilizadas.

[Table I - Description of the samples used.]

\begin{tabular}{cc}
\hline Amostra & Descrição \\
\hline VERM & vermiculita natural \\
VNa & vermiculita sódica \\
$\mathrm{Al}_{13} \mathrm{INT}$ & $\mathrm{Al}_{13}$-vermiculita intercalada \\
$\mathrm{Al}_{13} \mathrm{PILV}$ & $\mathrm{Al}_{13}$-vermiculita pilarizada \\
$\mathrm{LaAl}_{12} \mathrm{INT}$ & $\mathrm{LaAl}_{12}$-vermiculita intercalada \\
$\mathrm{LaAl}_{12} \mathrm{PILV}$ & $\mathrm{LaAl}_{12}$-vermiculita pilarizada \\
\hline
\end{tabular}

Métodos de caracterização

Difração de raios $X$ (DRX): os experimentos com difração de raios $X$ foram realizados em difratômetro Rigaku (DMAXB) de pó, com velocidade de varredura $0,5^{\circ} \min ^{-1}(2 \theta)$, radiação Cuk $\alpha \operatorname{com} 40 \mathrm{kV}$ e $25 \mathrm{~mA}$. Amostras com o tamanho de partícula inferior a $74 \mu \mathrm{m}$ (200 mesh) foram selecionadas.

Espectroscopia Mössbauer: espectros Mössbauer foram obtidos em geometria de transmissão padrão em um espectrômetro de aceleração constante com uma fonte radioativa de ${ }^{57} \mathrm{Co}$ em uma matriz de Rh. As medições foram efetuadas à temperatura ambiente em amostras em pó. Os espectros foram avaliados usando discretas funções lorentzianas de um conjunto de dubletos de quadrupolo e calculando a contribuição de cada curva para o espectro de absorção total do procedimento de mínimos quadrados do pacote de software Normos ${ }^{\circledR}$. Todos os deslocamentos isoméricos ( $\delta$ ) são mencionados em relação ao ferro metálico $(\alpha-\mathrm{Fe})$.

Ressonância magnética nuclear do estado sólido - ${ }^{27} \mathrm{Al}$ : os espectros de RMN ${ }^{27} \mathrm{Al}$ MAS foram registrados a 300,13 $\mathrm{MHz}$ num espectrômetro Bruker DRX-300 (7,05 T), operando a 78,2 MHz, utilizando uma seqüência de pulsos, a uma freqüência de amostragem de $10 \mathrm{kHz}$ a fiação, todas as medições foram efetuadas à temperatura ambiente; as análises foram conduzidas utilizando um pulso $\pi / 6$ curto (0,5 s), com 6000 acumulações e os deslocamentos químicos foram medidos em relação ao padrão $\mathrm{AlCl}_{3} \cdot 6 \mathrm{H}_{2} \mathrm{O}$.

Análise de BET (isotermas de $N_{2}$ ): as isotermas de adsorção de nitrogênio foram obtidas em temperatura de nitrogênio líquido, em um analisador de adsorção Micromeritics PAEA 2020C, no qual as amostras eram previamente calcinadas a $773 \mathrm{~K}$ (a menos que indicado de outra forma), e desgaseificada sob vácuo durante $12 \mathrm{~h}$ in situ a $573 \mathrm{~K}$. As áreas de superfície foram obtidas a partir do tratamento das isotermas BET na faixa de pressão relativa 0,05-0,25, e pelo método de Langmuir para as formas de pilarizadas. As superfícies externas e volumes de microporo das vermiculitas pilarizadas foram determinados após uma abordagem recentemente proposta. Os volumes totais de poros foram obtidos a partir da quantidade de nitrogênio adsorvido em $\mathrm{P} / \mathrm{P}_{0}=0,985$. Os volumes do microporo também foram determinados a partir do método t-plot [27].

\section{RESULTADOS E DISCUSSÃO}

A Fig. 1 mostra o difratograma da amostra de vermiculita natural (VERM), onde se pode evidenciar a presença de um pico em $7,12^{\circ}$ em $2 \theta$, possivelmente referente ao mineral interestratificado hidrobiotita, que pode ocorrer com a hidratação de flogopita/biotita por água de meteorização na superfície de rochas intemperizadas, durante a conversão para vermiculita [28].

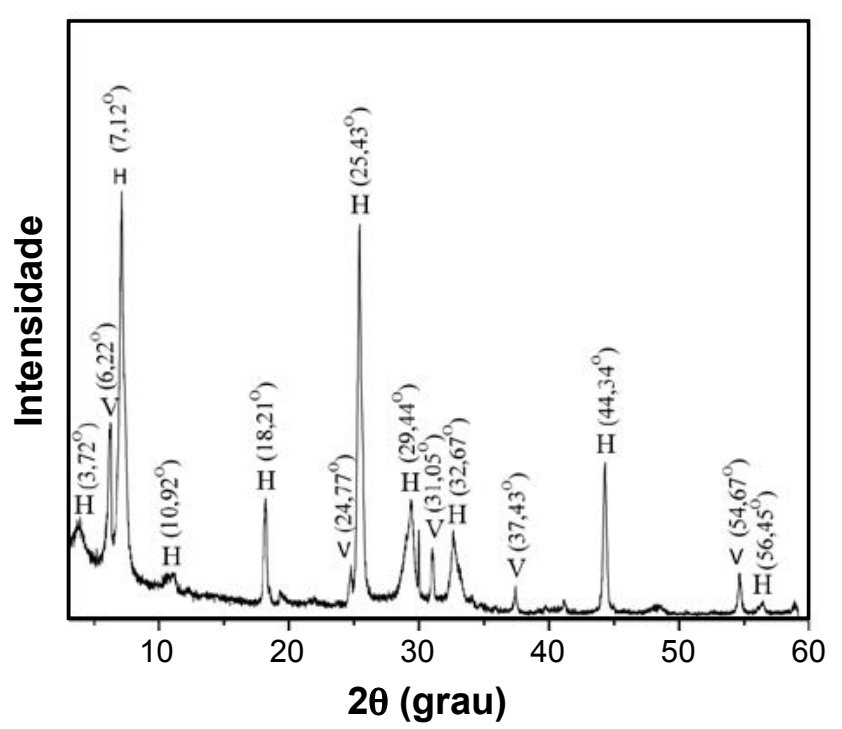

Figura 1: Padrões DRX da VERM: (V) vermiculita e $(\mathrm{H})$ hidrobiotita interistratificada.

[Figure 1: XRD patterns of RED: $(V)$ vermiculite and (H) interstratified hydrobiotite.] 


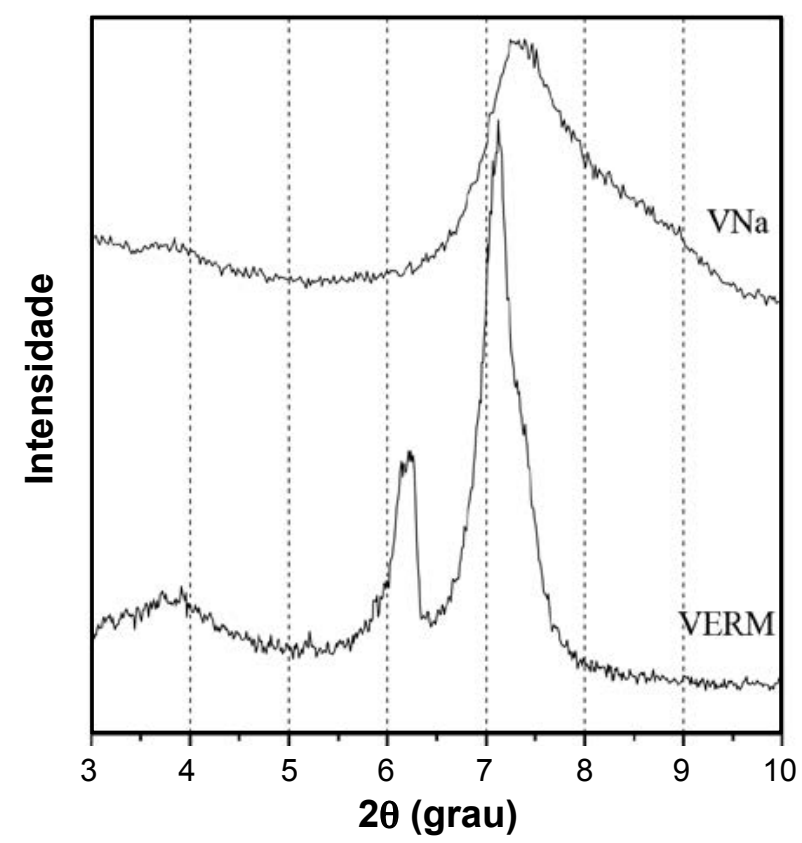

Figura 2: DRX das amostras VERM e VNa.

[Figure 2: XRD patterns of the samples VERM and VNa.]

Tabela II - Ângulo (20) e distância interplanar basal (d) das amostras em diferentes etapas.

[Table II - Angle (20) and basal interplanar distance (d) of the samples at different stages.]

\begin{tabular}{ccc}
\hline Amostra & $2 \theta$ (grau) & $\mathrm{d}(\mathrm{nm})$ \\
\hline $\mathrm{VNa}$ & 7,22 & 1,22 \\
$\mathrm{Al}_{13} \mathrm{INT}$ & 4,74 & 1,86 \\
$\mathrm{Al}_{13} \mathrm{PILV}$ & 4,96 & 1,78 \\
$\mathrm{LaAl}_{12} \mathrm{INT}$ & 4,69 & 1,88 \\
$\mathrm{LaAl}_{12} \mathrm{PILV}$ & 4,82 & 1,83 \\
\hline
\end{tabular}

O pico em $6,22^{\circ} 2 \theta$ com valor da distância interplanar basal de $\mathrm{d}=1,42 \mathrm{~nm}$ observado (Fig. 2), no difratograma da vermiculita natural (VERM) é correspondente ao pico característico desse tipo de mineral argiloso magnesiano [29].

A partir do tratamento com $\mathrm{HNO}_{3}$, seguido de calcinação a $873 \mathrm{~K}$ e remoção de ferro com ácido oxálico, (Fig. 2) ocorreu uma redução do valor da distância interplanar (d) de 1,42 nm para 1,22 nm, sendo assim uma possível comprovação do êxito na obtenção da amostra sódica (VNa) por troca iônica.

Os difratogramas a e b (Fig. 3) mostram que houve intercalação seguida de pilarização, onde se observa ainda uma leve contração estrutural devido à calcinação durante a pilarização, mesmo assim ambas as amostras demonstraram cristalinidade considerável. A amostra $\mathrm{Al}_{13}$ PILV (Fig. 3b) apresentou maior cristalinidade em relação a $\mathrm{LaAl}_{12} \mathrm{PILV}$ (Fig. 3d). A intensidade difratada de qualquer reflexão hkl a partir de qualquer material cristalino pode estar relacionada à sua composição, matriz e as características texturais [30].

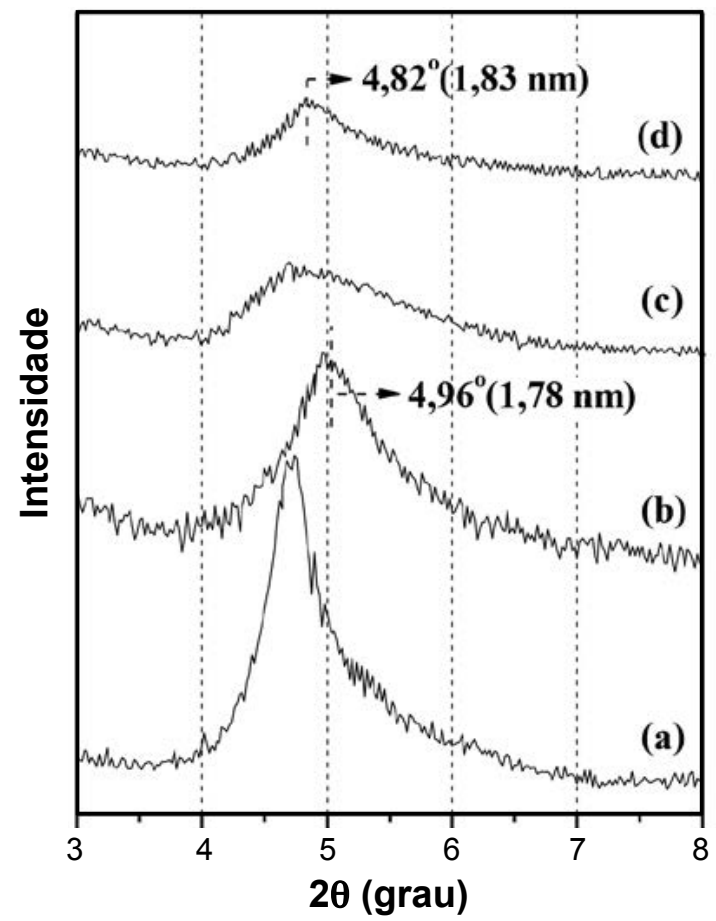

Figura 3: DRX das amostras: (a) Al13INT, (b) Al13PILV, (c) LaA112INT e (d) LaA112PILV.

[Figure 3: XRD patterns of samples: (a) Al13INT, (b) All3PILV, (c) LaAl12INT and (d) LaAl12PILV.]

Os difratogramas b e d (Fig. 3) comprovam que houve um aumento da distância interplanar basal entre as amostras modificadas com os oligômeros $\mathrm{Al}_{13}$ e $\mathrm{LaAl}_{12}$, sendo a última a que apresentou maior distância interplanar basal, possivelmente devido ao maior raio iônico do lantânio, que é de 117,2 pm em comparação com o do alumínio que é de 53,5 pm, tendo assim formado um policátion com características estéricas maiores.

Os resultados (parâmetros hiperfinos) obtidos através do ajuste dos espectros Mössbauer são apresentados na Tabela II. Os espectros Mössbauer (Fig. 4) foram obtidos a partir de amostras na forma de pó da argila natural (VERM), sódica $(\mathrm{VNa})$, intercalada $\left(\mathrm{Al}_{13} \mathrm{INT}\right.$ e $\left.\mathrm{LaAl}_{12} \mathrm{INT}\right)$ e pilarizada $\left(\mathrm{Al}_{13} \mathrm{PILV}\right.$ e $\mathrm{LaAl}_{12} \mathrm{PILV}$ ) com alumínio e alumínio-lantânio.

Tabela III - Parâmetros hiperfinos das diferentes amostras de vermiculita.

[Table III - hyperfine parameters of different samples of vermiculite.]

\begin{tabular}{ccccc}
\hline Sample & Sítio & $\begin{array}{c}\delta \\
\left(\mathrm{mm} \cdot \mathrm{s}^{-1}\right)\end{array}$ & $\begin{array}{c}\Delta \mathrm{E}_{\mathrm{Q}} \\
\left(\mathrm{mm} \cdot \mathrm{s}^{-1}\right)\end{array}$ & $\begin{array}{c}\Gamma \\
\left(\mathrm{mm} \cdot \mathrm{s}^{-1}\right)\end{array}$ \\
\hline $\mathrm{VERM}$ & $\mathrm{Fe}^{3+}$ & 0,34 & 0,89 & 0,71 \\
$\mathrm{VNa}$ & $\mathrm{Fe}^{2+}$ & 1,28 & 2,33 & 0,37 \\
$\mathrm{Fe}^{3+}$ & 0,37 & 1,20 & 0,65 \\
$\mathrm{Al}_{13} \mathrm{INT}$ & $\mathrm{Fe}^{3+}$ & 0,37 & 1,19 & 0,65 \\
$\mathrm{Al}_{13} \mathrm{PILV}$ & $\mathrm{Fe}^{3+}$ & 0,37 & $1,28^{*}$ & 0,70 \\
$\mathrm{LaAl}_{12} \mathrm{INT}$ & $\mathrm{Fe}^{3+}$ & 0,38 & 1,18 & 0,66 \\
$\mathrm{LaAl}_{12} \mathrm{PILV}$ & $\mathrm{Fe}^{3+}$ & 0,38 & $1,21^{*}$ & 0,67 \\
\hline
\end{tabular}




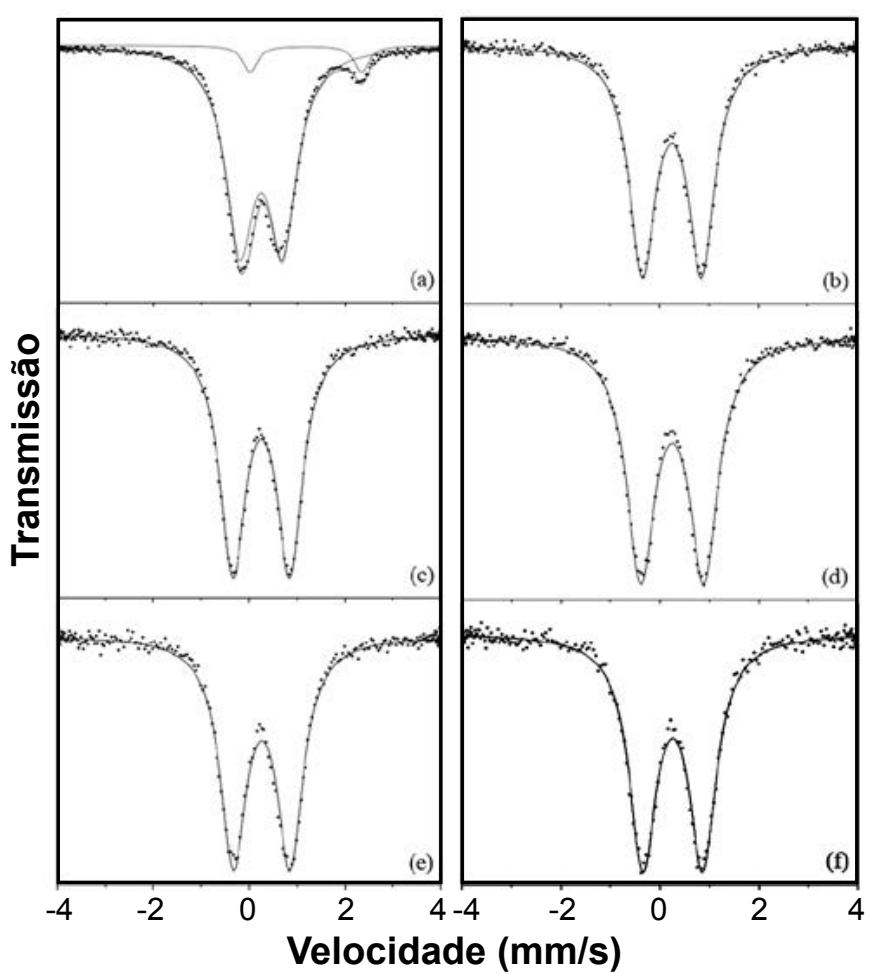

Figura 4: Espectros Mössbauer do Fe: (a) VERM, (b) VNa, (c) $\mathrm{Al}_{13}$ INT, (d) $\mathrm{Al}_{13} \mathrm{PILV}$, (e) $\mathrm{LaAl}_{12} \mathrm{INT}$ e (f) $\mathrm{LaAl}_{12} \mathrm{PILV}$.

[Figure 4: Mössbauer spectra of $\mathrm{Fe}$ : (a) VERM, (b) VNa, (c) $A l_{13} I N T$, (d) $A l_{13} P I L V$, (e) LaAl ${ }_{12} I N T$ and (f) LaAl $\left.{ }_{12} P I L V.\right]$

Todos mostram a presença de ferro no estado de oxidação $\mathrm{Fe}^{3+}$. A Tabela III mostra os parâmetros hiperfinos, onde se pode comprovar a inexistência de $\mathrm{Fe}^{2+}$ nas amostras de vermiculita diferentes da natural, o que pode ter sido causado pelo tratamento químico realizado nessas amostras.

As amostras pilarizadas (Tabela III), mostraram valores de desdobramento quadrupolar $(\Delta)$ consideravelmente diferentes $*\left(1,28\right.$ e $\left.1,21 \mathrm{~mm} \cdot \mathrm{s}^{-1}\right)$, respectivamente, esses valores decrescentes podem estar relacionados com o aumento na organização do sítio octaédrico do ferro.

Acredita-se que tal aumento de simetria do ferro, está relacionado com a redução das tensões experimentadas pelas folhas octaédricas quando os pilares de óxido são formados, devido possivelmente ao encurtamento das ligações Fe-O.

Podem ocorrer pequenas variações nos parâmetros $\delta \mathrm{e}$ $\Delta \mathrm{E}_{\mathrm{O}}$, especialmente se considerar que o ambiente químico do $\mathrm{Fe}^{3+}$ na estrutura do mineral pode variar ligeiramente, e distorções na geometria dos sítios de $\mathrm{Fe}^{3+}$ devido à perda de água ou de grupos $\mathrm{OH}$ na etapa de calcinação [31]. A intercalação dos complexos $\left[\mathrm{Al}_{13} \mathrm{O}_{4}(\mathrm{OH})_{24}\left(\mathrm{H}_{2} \mathrm{O}\right)_{12}\right]^{7+}$ e $\left[\mathrm{LaAl}_{12} \mathrm{O}_{4}(\mathrm{OH})_{24}\left(\mathrm{H}_{2} \mathrm{O}\right)_{12}\right]^{7+}$ causou aumento da intensidade do sinal de Al octaédrico, como esperado. Com a calcinação ocorreu uma diminuição da intensidade do sinal, relativo à perda de hidroxilas e águas de coordenação, acarretando na geração de sítios de Al tetraédrico.

A Tabela IV mostra os valores de deslocamento químico

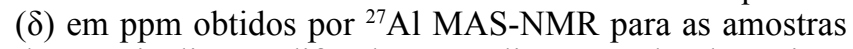
de vermiculita modificada com oligômero de alumínio e alumínio-lantânio. A vermiculita de partida apresentou (Tabela IV) predominância de $\mathrm{Al}$ tetraédricos e um pequeno sinal na região de $\mathrm{Al}$ octaédrico.

Tabela IV - Resultados de deslocamentos químicos e áreas obtidos nos espectros de RMN-MAS de ${ }^{27} \mathrm{Al}$.

[Table IV-Results of areas and chemical shifts obtained in the NMR-MAS spectra of ${ }^{27} \mathrm{Al}$.]

\begin{tabular}{cclc}
\hline Amostra & $(\delta$, ppm) & Assinalamento & $\begin{array}{c}\text { Área relativa } \\
(\%)\end{array}$ \\
\hline VERM & 9,6 & $\mathrm{Al}^{\mathrm{VI}}$ & 6,4 \\
& 54,2 & $\mathrm{Al}^{\mathrm{IV}}$ & 93,6 \\
$\mathrm{Al}_{13} \mathrm{INT}$ & 1,6 & $\mathrm{Al}^{\mathrm{VI}}$ & 66,0 \\
& 59,8 & $\mathrm{Al}^{\mathrm{IV}}$ & 34,0 \\
$\mathrm{Al}_{13} \mathrm{PILV}$ & 4,4 & $\mathrm{Al}^{\mathrm{VI}}$ & 48,4 \\
& 55,4 & $\mathrm{Al}^{\mathrm{IV}}$ & 51,6 \\
$\mathrm{LaAl}_{12} \mathrm{INT}$ & 3,0 & $\mathrm{Al}^{\mathrm{VI}}$ & 44,8 \\
& 55,9 & $\mathrm{Al}^{\mathrm{IV}}$ & 55,2 \\
$\mathrm{LaAl}_{12} \mathrm{PILV}$ & 3,7 & $\mathrm{Al}^{\mathrm{VI}}$ & 71,7 \\
& 56,0 & $\mathrm{Al}^{\mathrm{IV}}$ & 28,3 \\
\hline
\end{tabular}

A Fig. 5 mostra as isotermas de adsorção/dessorção de $\mathrm{N}_{2}$ a 77 K, das amostras sem e com lantânio, respectivamente, onde se observa que a isoterma (5a), se trata de uma isoterma do tipo IV, típico de adsorvente mesoporoso. $\mathrm{Na}$ curva de adsorção/dessorção da vermiculita (Fig. 5a), se observa histerese, que se deve possivelmente ao fato da vermiculita ser metaestável. A amostra VERM não é um material com grandes tamanhos de poros, e antes dos tratamentos apresentou área superficial específica, volume total de microporos e diâmetros de poro consideravelmente baixos (Tabela IV).

A histerese observada tanto na amostra (b) quando na amostra (c) estão associadas a condensação capilar nos mesoporos [32]. Surpreendentemente os perfis das curvas são muito parecidos, como o raio de van der Walls do lantânio supera consideravelmente o do alumínio, se esperaria o surgimento de desordem na microestrutura do pilar. O valor de diâmetro de poro observado para a amostra $\mathrm{LaAl}_{12} \mathrm{PILV}$ (Tabela V), reflete seu maior tamanho passou de 3\% sugere que o lantânio encontra-se em sítios tetraédricos.

Tabela V - Características texturais por adsorção de nitrogênio obtidas para diferentes amostras de vermiculita.

[Table $V$ - Textural features for nitrogen adsorption obtained for different samples of vermiculite.]

\begin{tabular}{ccccc}
\hline Amostra & $\begin{array}{c}\mathrm{S}_{\mathrm{BET}} \\
\left(\mathrm{m}^{2} \cdot \mathrm{g}^{-1}\right)\end{array}$ & $\begin{array}{c}\mathrm{S}_{\text {LANGMUR }} \\
\left(\mathrm{m}^{2} \cdot \mathrm{g}^{-1}\right)\end{array}$ & $\begin{array}{c}\mathrm{V}_{\mu} \\
\left(\mathrm{cm}^{3} \cdot \mathrm{g}^{-1}\right)\end{array}$ & $\begin{array}{c}\mathrm{D}_{\mathrm{p}} \\
(\mathrm{nm})\end{array}$ \\
\hline VERM & 6,1 & 7,8 & 0,03 & 1,73 \\
$\mathrm{Al}_{13}$ PILV & 211,6 & 310,0 & 120,8 & 3,75 \\
$\mathrm{LaAl}_{12}$ PILV & 178,4 & 264,1 & 81,6 & 3,85 \\
\hline
\end{tabular}




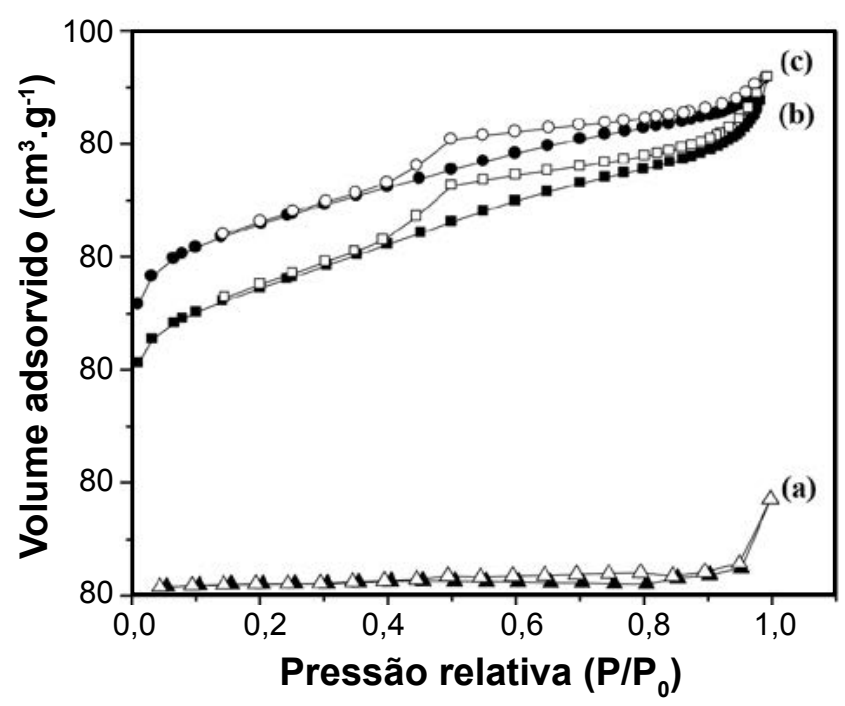

Figura 5: Isotermas de adsorção e dessorção de $\mathrm{N}_{2}$ a $77 \mathrm{~K}$ das amostras: (a) VERM, (b) LaAl ${ }_{12}$ PILV e (c) $\mathrm{Al}_{13}$ PILV.

[Figure 5: Adsorption and desorption isotherms of $N_{2}$ at $77 \mathrm{~K}$ of samples: (a) VERM, (b) LaAl ${ }_{12}$ PILV and (c) $\mathrm{Al}_{13}$ PILV.]

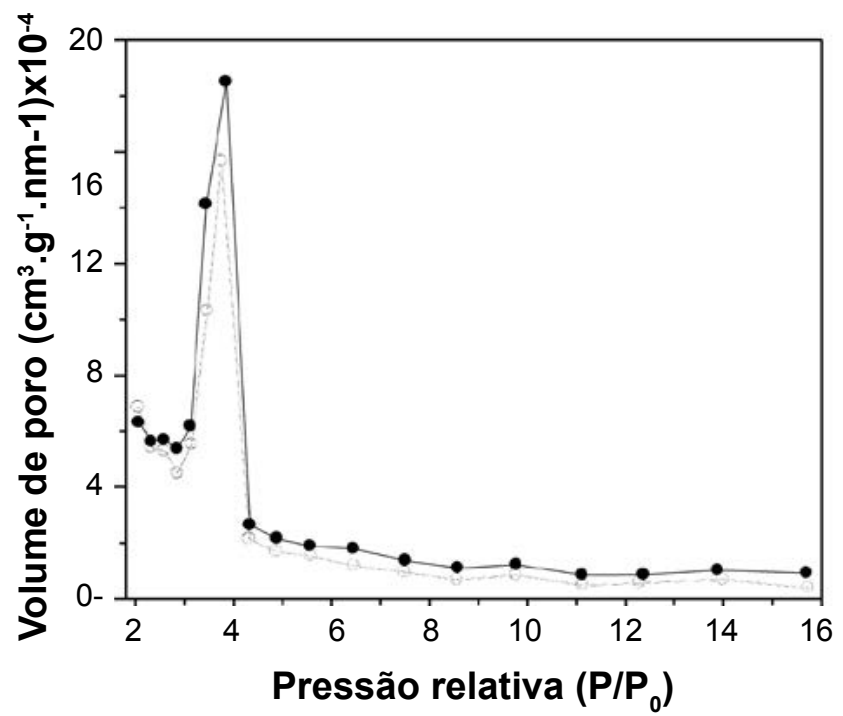

Figura 6: Distribuição de diâmetro de poro da vermiculita pilarizada com alumínio Al13PILV (-o) e com alumínio-lantânio LaAl12PILV $(\rightarrow-)$.

[Figure 6: Distribution of pore diameter of the aluminum-pillared vermiculite Al13PILV (-o) and lanthanum aluminum LaAl12PILV $(\bullet)$.

Apesar da evidência de existência de lantânio na estrutura do pilar, a pequena diferença sugere não ter ocorrido à formação de uma estrutura oligomérica com o lantânio ao centro, como o indicado pelas proporções relativas de $\mathrm{Al}^{\mathrm{VI}}$ e $\mathrm{Al}^{\mathrm{IV}}$.

A isoterma (5b) da amostra $\mathrm{LaAl}_{12} \mathrm{PILV}$, apresentou distribuição de poros por dessorção BJH (Fig. 6), referente ao procedimento visando o aumento do diâmetro de poro através da intercalação com sal de alumínio dopado com lantânio, resultou em aumento significativo das características textuais (Tabela V), em comparação com os observados na amostra natural sem nenhum tratamento químico.

\section{CONCLUSÕES}

Em comparação os procedimentos de intercalação e pilarização com os intercalantes $\mathrm{Al}_{13} \mathrm{e} \mathrm{LaAl}_{12}$, para obtenção das amostras de vermiculita mesoporosa mostraram características distintas. As análises de características texturais, (BET) e de distribuição de poros (BJH), mostraram que a amostra VERM apresentou valores extremamente baixos de área superficial e diâmetro de poro, portanto sem relevância para os propósitos desta pesquisa, e que a amostra $\mathrm{Al}_{13}$ PILV apresentou maior área superficial em relação à amostra $\mathrm{LaAl}_{12} \mathrm{PILV}$, sendo que ambas possuem diâmetro de poro com valores próximos, confirmando assim a relevância de todo o processo de modificação química até a obtenção das amostras de argilas mesoporosas. A partir dos resultados de espectroscopia Mössbauer, conclui-se que existe uma relação entre aumento da simetria do sítio octaédrico do ferro $\left(\mathrm{Fe}^{3+}\right)$ e aumento da dopagem com lantânio, podendose inferir que quanto mais simétrico for o sítio octaédrico, maior será a acidez. Os resultados de RMN do estado sólido $\left({ }^{27} \mathrm{Al}\right)$ obtidos, evidenciaram que a amostra de vermiculita natural (VERM) não possui valores consideráveis de alumínio hexacoordenado, predominando a existência de alumínio tetracoordenado. Conclui-se ainda que ambas as amostras intercaladas e calcinadas apresentaram valores consideráveis de alumínio hexacoordenado, sendo esses maiores ainda após a dopagem com lantânio do oligômero de alumínio, o que estar condizente com o fato de que o oligômero possui mais $\mathrm{Al}$ octaédrico que tetraédrico, gerando assim uma argila modificada com quantidade relevante de $\mathrm{Al}$ hexacoordenado em sua estrutura.

\section{AGRADECIMENTOS}

À Prof. R. San Gil (Universidade Federal do Rio de Janeiro) pelas análises para obtenção dos espectros de RMN do estado sólido e o suporte financeiro do CNPq e da CAPES.

\section{REFERÊNCIAS}

[1] B. K. Ngun, H. Mohamad, S. K. Sulaiman, K. Okada, Z. A. Ahmad, Appl. Clay Sci. 53 (2011) 33-41.

[2] L. Jin, B. Dai, Appl. Surface Sci. 258 (2012) 3386-3392.

[3] G. Abate, L. B. O. dos Santos, S. M. Colombo, J. C. Masini, Appl. Clay Sci. 32 (2006) 261-270.

[4] M. Kehal, L. Reinert, L. Duclaux, Appl. Clay Sci. 48 (2010) 561-568.

[5] D. Malferrari, M. F. Brigatti, A. Marcelli, W. Chu, Z. Wu, Appl. Clay Sci. 50 (2010) 12-18.

[6] M. C. Jiménez de Haro, J. L. Pérez-Rodríguez, J. Poyato, L. A. Pérez-Maqueda, V. Ramírez-Valle, A. Justo, A. Lerf, F. E. Wagner, Appl. Clay Sci. 30 (2005) 11-20.

[7] Q. Chen, P. Wu, Z. Dang, N. Zhu, P. Li, J. Wu, X. Wang, Sep. Purif. Technol. 71 (2010) 315-323.

[8] E. V. D. Gomes, L. L. Y. Visconte, E. B. A. V. Pacheco, Cerâmica 56, 337 (2010) 44-48. 
[9] K. Okada, N. Arimitsu, Y. Kameshima, A. Nakajima, K. J. D. MacKenzie, Appl. Clay Sci. 31 (2006) 185-193. [10] M. Zhao, Z. Tang, P. Liu, J. Hazard. Mater. 158 (2008) 43-51.

[11] C. Maqueda, J. L. Perez-Rodriguez, J. Šubrt, N. Murafa, Appl. Clay Sci. 44 (2009) 178-184.

[12] C. Maqueda, A. S. Romero, E. Morillo, J. L. PerezRodriguez, J. Phys. Chem. Solids 68 (2007) 1220-1224.

[13] L. Wang, Z. Chen, X. Wang, S. Yan, J. Wang, Y. Fan, Appl. Clay Sci. 51 (2011) 151-157.

[14] S. A. Suvorov, V. V. Skurikhin, Refrac. Ind. Ceram. 44 (2003) 186-193.

[15] M. R. M. P. de Aguiar, A. C. Novaes, Quím. Nova 25 (2005) 1145-1154.

[16] D. Nguyen-Thanh, T. J. Bandosz, Micro. Meso. Mater. 92 (2006) 47-55.

[17] J. Pires, A. C. Araújo, A. P. Carvalho, M. L. Pinto, J. M. González-Calbet, J. Ramírez-Castellanos, Micro. Meso. Mater. 73 (2004) 175-180.

[18] K. Sapag, S. Mendioroz, Synthesis and characterization of micro-mesoporous solids: pillared clays, Colloids Surf. A. 187-188 (2001) 141-149.

[19] C. Volzone, Appl. Clay Sci. 36 (2007) 191-196.

[20] Wen-Tien Tsai, Ting-Yi Su, Hsin-Chieh Hsu, Keng-Yu Lin, Chien-Ming Lin, Tsung-Hung Tai, Micro. Meso. Mater. 102 (2007) 196-203.

[21] T. Mishra, P. Mohapatra, K. M. Parida, Appl. Catal. B.
79 (2008) 279-285.

[22] M. Altunlu, S. Yapar, Colloids Surf. A. 306 (2007) 8894.

[23] N. R. Sanabria, M. A. Centeno, R. Molina, S. Moreno, Appl. Catal. A. 356 (2009) 243-249.

[24] A. Gil, F. C. C. Assis, S. Chem. Eng. J. 168 (2011) 1032-1040.

[25] A. Gil, M. A. Vicente, S. A. Korili, J. Catal. 229 (2005) 119-126.

[26] F. del Rey-Perez-Caballero, G. Micro. Meso. Mater. 41 (2000) 169-181.

[27] Micromeritics, Operator's Manual - ASAP 2020 - Accelerated Surface Area and Porosimetry System, Micromeritics Instr. Corp. V3.01 (2004-2006) 22.

[28] H. F. Muiambo, W. W. Focke, M. Atanasova, I. van der Westhuizen, L. R. Tiedt, Appl. Clay Sci. 50 (2010) 51-57.

[29] V. Mate jka, M. Šupová, V. Klemm, D. Rafaja, M. Valášková, J. Tokarsky', J. Lešková, E. Plevová, Micro. Meso. Mater. 129 (2010) 118-125.

[30] V. R. Ouhadi, R. N. Yong, Appl. Clay Sci. 23 (2003) 141-148.

[31] J. G. Carriazo, M. A. Centeno, J. A. Odriozola, S. Moreno, R. Molina, Appl. Catal. A. 317 (2007) 120-128.

[32] K. S. W. Sing, D. H. Everett, R. A. W. Haul, L. Moscou, R. A. Perotti, J. Rouquérol, T. Siemieniewska, Pure Appl. Chem. 57 (1985) 603-619.

(Rec. 20/05/2013, Ac. 16/11/2013) 